\title{
Formation of giant globular cluster G1 and the origin of the M 31 stellar halo
}

\author{
K. Bekki ${ }^{1}$ and M. Chiba ${ }^{2}$ \\ ${ }^{1}$ School of Physics, University of New South Wales, Sydney 2052, Australia \\ 2 Astronomical Institute, Tohoku University, Sendai, 980-8578, Japan \\ e-mail: chiba@astr.tohoku.ac.jp
}

Received 20 September 2003 / Accepted 21 October 2003

\begin{abstract}
We demonstrate that globular cluster G1 could have been formed by tidal interaction between M 31 and a nucleated dwarf galaxy (dE,N). Our fully self-consistent numerical simulations show that during tidal interaction between M 31 and G1's progenitor dE,N with $M_{B} \sim-15$ mag and its nucleus mass of $\sim 10^{7} M_{\odot}$, the dark matter and the outer stellar envelope of the $\mathrm{dE}, \mathrm{N}$ are nearly completely stripped whereas the nucleus can survive the tidal stripping because of its initially compact nature. The naked nucleus (i.e., G1) has orbital properties similar to those of its progenitor dE,N. The stripped stars form a metal-poor $([\mathrm{Fe} / \mathrm{H}] \sim-1)$ stellar halo around M 31 and its structure and kinematics depend strongly on the initial orbit of G1's progenitor $\mathrm{dE}, \mathrm{N}$. We suggest that the observed large projected distance of G1 from M 31 ( $\sim 40 \mathrm{kpc}$ ) can give some strong constraints on the central density of the dark matter halo of dE,N. We discuss these results in the context of substructures of M 31's stellar halo recently revealed by Ferguson et al. (2002).
\end{abstract}

Key words. galaxies: halos - galaxies: individual: M 31 - galaxies: interactions - galaxies: star clusters Galaxy: globular clusters: individual: $\omega$ Centauri - Galaxy: globular clusters: individual: Mayall II = G1

\section{Introduction}

A growing number of photometric and spectroscopic observations have suggested that G1 (=Mayall II), which is one of the brightest globular clusters belonging to M 31, has very unique physical properties as a globular cluster (e.g., Meylan et al. 2001). These include a possible intrinsic metallicity dispersion among its stellar population (Meylan et al. 2001), the large central velocity dispersion of $\sim 25 \mathrm{~km} \mathrm{~s}^{-1}$ (e.g., Djorgovski et al. 1997), the very flattened shape with mean ellipticity of 0.2 and significantly high central surface brightness (Rich et al. 1996; Meylan et al. 2001), and the possible existence of a black hole with a mass of $20000 M_{\odot}$ (Gebhardt et al. 2002). In spite of its extraordinary nature, $\mathrm{G} 1$ is observed to be on the $M_{V}-\sigma_{0}$ relation (where $M_{V}$ and $\sigma_{0}$ are total magnitude in $V$-band and central velocity dispersion) defined not by elliptical and dwarf galaxies but by globular clusters, which implies that G1 looks like a genuine globular cluster (Meylan et al. 2001).

One of the possible scenarios of G1 formation is that $\mathrm{G} 1$ is the surviving nucleus of an ancient nucleated dwarf galaxy with its outer stellar envelope almost entirely stripped by M 31's strong tidal field (Meylan et al. 1997, 2000, 2001). Such a scenario has already been suggested by Zinnecker et al. (1988) and Freeman (1993), and the viability of the scenario has been extensively discussed by many authors in terms of $\omega$ Cen

Send offprint requests to: $\mathrm{K}$. Bekki,

e-mail: bekki@bat.phys.unsw.edu.au formation (e.g., Hilker \& Richtler 2000; Dinescu 2002; Gnedin et al. 2002; Zhao 2002; Bekki \& Freeman 2003; Mizutani et al. 2003). However, no theoretical attempts have been made so far to investigate (1) whether M 31's tidal filed is strong enough to transform a dE,N into G1 and (2) what observable evidence of the past destruction of G1's progenitor $\mathrm{dE}, \mathrm{N}$ we can find in the M 31 halo regions. The above point (2) is very important, because Ibata et al. (2001) and Ferguson et al. (2002) have recently discovered M 31's stellar halo substructures, which could have been formed from tidal destruction of M 31's satellite dwarfs.

In this paper, by using numerical simulations, we first demonstrate that $\mathrm{G} 1$ can be formed from a dE, N during tidal interaction between the dE,N and M 31. Our fully self-consistent numerical simulations demonstrate that the stellar envelope of dE,N with $M_{B} \sim-15$ can be nearly completely stripped by the strong tidal field of M 31 whereas the central nucleus can remain intact owing to its compactness. We suggest that this naked nucleus orbiting M 31 is a giant globular cluster (i.e., G1). The morphological transformation from dE,Ns into very compact stellar systems was originally investigated by Bekki et al. (2001, 2003) for ultra-compact dwarfs labeled as "UCD" (Drinkwater et al. 2003) and called "galaxy threshing" (Bekki et al. 2001), though they suggested that galaxy threshing is also important for the formation of giant globular clusters such as $\omega$ Cen and G1. The present study confirms this early suggestion 
and discusses the physical relationship between UCDs, $\omega$ Cen, and G1.

\section{Model}

Since our numerical methods and techniques for modeling collisionless, self-gravitating systems of dE,Ns already have been described in detail by Bekki et al. (2003), we give only a brief review here. We consider a $\mathrm{dE}, \mathrm{N}$ with a mass and size similar to that observed for the dE,N types orbiting $M$ 31. The dE,N is modeled as a fully self-gravitating system and is assumed to consist of a dark matter halo, a stellar component and a nucleus: we first investigate this "three-component" dE,N model. For convenience, the stellar component (i.e., the main baryonic component) is referred to as either the "envelope" or the "stellar envelope" so that we can distinguish this component from the stellar nucleus. The density profile of the dark matter halo with the total mass of $M_{\mathrm{dm}}$ in the dE, $\mathrm{N}$ is represented by that proposed by Salucci \& Burkert (2000):

$\rho_{\mathrm{dm}}(r)=\frac{\rho_{\mathrm{dm}, 0}}{\left(r+a_{\mathrm{dm}}\right)\left(r^{2}+a_{\mathrm{dm}}\right)^{2}}$,

where $\rho_{\mathrm{dm}, 0}$ and $a_{\mathrm{dm}}$ are the central dark matter density and the core (scale) radius, respectively. This model profile is consistent with observations and different from the predictions of the standard CDM model (Navarro et al. 1996, hereafter NFW). The dark matter core parameters, $\rho_{\mathrm{dm}, 0}, a_{\mathrm{dm}}$, and $M_{0}$ (where $M_{0}$ is the total dark matter mass within $a_{\mathrm{dm}}$ ) have a clear observed correlation, $M_{0}=4.3 \times 10^{7}\left(\frac{a_{\mathrm{dm}}}{\mathrm{kpc}}\right)^{7 / 3} M_{\odot}$ (Burkert 1995).

The mass (luminosity) and the scale length of the stellar envelope of the $\mathrm{dE}, \mathrm{N}$ is modeled according to the observed scaling relation of Ferguson \& Binggeli (1994):

$\log a_{\mathrm{dw}}[\mathrm{pc}]=-0.02 M_{B}+2.6$,

for faint dwarfs $\left(M_{B} \geq-16\right)$, where $a_{\mathrm{dw}}$ and $M_{B}$ are the scale length of the exponential profile and the absolute $B$-band magnitude, respectively. The projected density of the envelope with $M_{B}$ and the total mass of $M_{\mathrm{dw}}$ (and $M_{\mathrm{dw}} / L_{B}=2$ ) is represented by an exponential profile with a scale length $a_{\mathrm{dw}}$. The projected density profile of the nucleus with mass $M_{\mathrm{n}}$ is represented by a King model (King 1964) with a core radius of $a_{\mathrm{n}}$ and a central concentration parameter $c$ of 1.0.

The nuclei typically contribute about a few percent of the total light of dwarfs (Binggeli \& Cameron 1991; Freeman 1993 ) and the present-day mass of G1 is estimated to be $1.5 \times$ $10^{7} M_{\odot}$ for the King model (Meylan et al. 2001). Considering these observations, the reasonable $M_{B}$ of dE,N is estimated to be -15 mag. Given a value of $M_{\mathrm{dm}} / M_{\mathrm{dw}}$, we can determine $a_{\mathrm{dm}}$ from the above $M_{0}-a_{\mathrm{dm}}$ relation (Burkert 1995). For convenience, $a_{\mathrm{dm}}$ for $M_{B}=-15 \mathrm{mag}$ and $M_{\mathrm{dm}} / M_{\mathrm{dw}}=5$ is referred to as $a_{\mathrm{dm}, 0}$ hereafter. We mainly investigate the dE,N models with $M_{B}=-15 \mathrm{mag}, a_{\mathrm{dw}}=790 \mathrm{pc}, M_{\mathrm{dm}} / M_{\mathrm{dw}}=5, a_{\mathrm{dm}}=a_{\mathrm{dm}, 0}$, $M_{\mathrm{n}} / M_{\mathrm{dw}}=0.05$, and $a_{\mathrm{n}} / a_{\mathrm{dw}}=0.02$. All of these values are reasonably consistent with observations (Binggeli \& Cameron 1991; Ferguson \& Binggeli 1994). We also investigate the models with $a_{\mathrm{dm}}=0.25 a_{\mathrm{dm}, 0}$ to clarify the importance of the dark matter halo structure of a dE,N in the formation processes of G1.
Table 1. Model parameters.

\begin{tabular}{lllll}
\hline \hline Model & $a_{\mathrm{dm}} / a_{\mathrm{dm}, 0}$ & $\theta($ degrees $)$ & $R_{\mathrm{apo}}(\mathrm{kpc})$ & $e_{\mathrm{p}}$ \\
\hline Fiducial & 1.0 & 30 & 80 & 0.62 \\
Smaller $e_{\mathrm{p}}$ & 1.0 & 30 & 80 & 0.18 \\
Smaller $R_{\text {apo }}$ & 1.0 & 30 & 40 & 0.62 \\
More compact dark matter & 0.25 & 30 & 80 & 0.62 \\
\hline
\end{tabular}

M 31 is assumed to have the disk mass of $7.8 \times 10^{10} M_{\odot}$, and the bulge-to-disk-ratio of 0.25 , and the maximum rotational velocity of $260 \mathrm{~km} \mathrm{~s}^{-1}$, all of which are consistent with observations (e.g., van den Bergh 2000). The initial disk plane of M 31 is set to be the $x-y$ plane of a simulation. The dE,N orbiting M 31 has the initial position of $(x, y, z)=\left(R_{\text {apo }} \cos \theta\right.$, $\left.0, R_{\text {apo }} \sin \theta\right)$ and the initial velocity of $\left(v_{x}, v_{y}, v_{z}\right)=\left(0, \alpha V_{\mathrm{c}}\right.$, 0 ), where $R_{\text {apo }}, \theta$, and $V_{\mathrm{c}}$ are the apocenter of the orbit, the inclination angle with respect to the M 31's disk (i.e., the $x-y$ plane), the circular velocity at the apocenter, and the parameter $(0 \leq \alpha \leq 1)$ that determines the orbital eccentricity represented by $e_{\mathrm{p}}$ (i.e., the larger $\alpha$ is, the more circular the orbit is). G1 is observed to have the projected distance of $40 \mathrm{kpc}$ from $\mathrm{M} 31$ and radial velocity of $-31 \mathrm{~km} \mathrm{~s}^{-1}$ with respect to M 31 (e.g., Meylan et al. 2001). Guided by these observations, we investigate the models with $\theta=30^{\circ}$ and $80^{\circ}, R_{\text {apo }}=40,80$, and $160 \mathrm{kpc}$, and $e_{\mathrm{p}}=0.62(\alpha=0.5)$ and $0.18(\alpha=0.9)$. We however describe the four representative and important models in this paper and the parameter values in these models (e.g., "fiducial model") are given in Table 1. All the simulations have been carried out on a GRAPE board (Sugimoto et al. 1990) with the particle number of 90000 .

\section{Results}

Figures 1 summarizes the morphological evolution of the envelope and the nucleus of the $\mathrm{dE}, \mathrm{N}$ in the fiducial model, which shows typical behavior in morphological transformation from a dE,N into G1. As the dE,N approaches the pericenter of its orbit, the strong global tidal field of M 31 stretches the envelope of the $\mathrm{dE}, \mathrm{N}$ along the direction of the dwarf's orbit and consequently tidally strips the stars of the envelope $(T=0.6 \mathrm{Gyr})$. The dark matter halo, which is more widely distributed than the envelope due to its larger core radius, is also efficiently removed from the dE,N during the pericenter passage. Since the envelope (and the dark matter halo) loses a significant fraction of its mass during the passage through the pericenter, the envelope becomes more susceptible to the tidal effects of M 31 after the pericenter passage. Therefore, each subsequent time the dwarf approaches the pericenter, it loses an increasingly larger fraction of its stellar envelope through tidal stripping. Finally, the envelope and the dark matter halo lose $98 \%$ and $99 \%$ of their initial masses, respectively, after four passages through the pericenter ( $T=4.8 \mathrm{Gyr})$.

The central nucleus, on the other hand, is only weakly influenced by the tidal force as a result of its compact configuration. Because of its strongly self-gravitating nature, the nucleus loses only a small amount $(\sim 7 \%)$ of its mass and thus maintains its compact morphology during its tidal interaction 

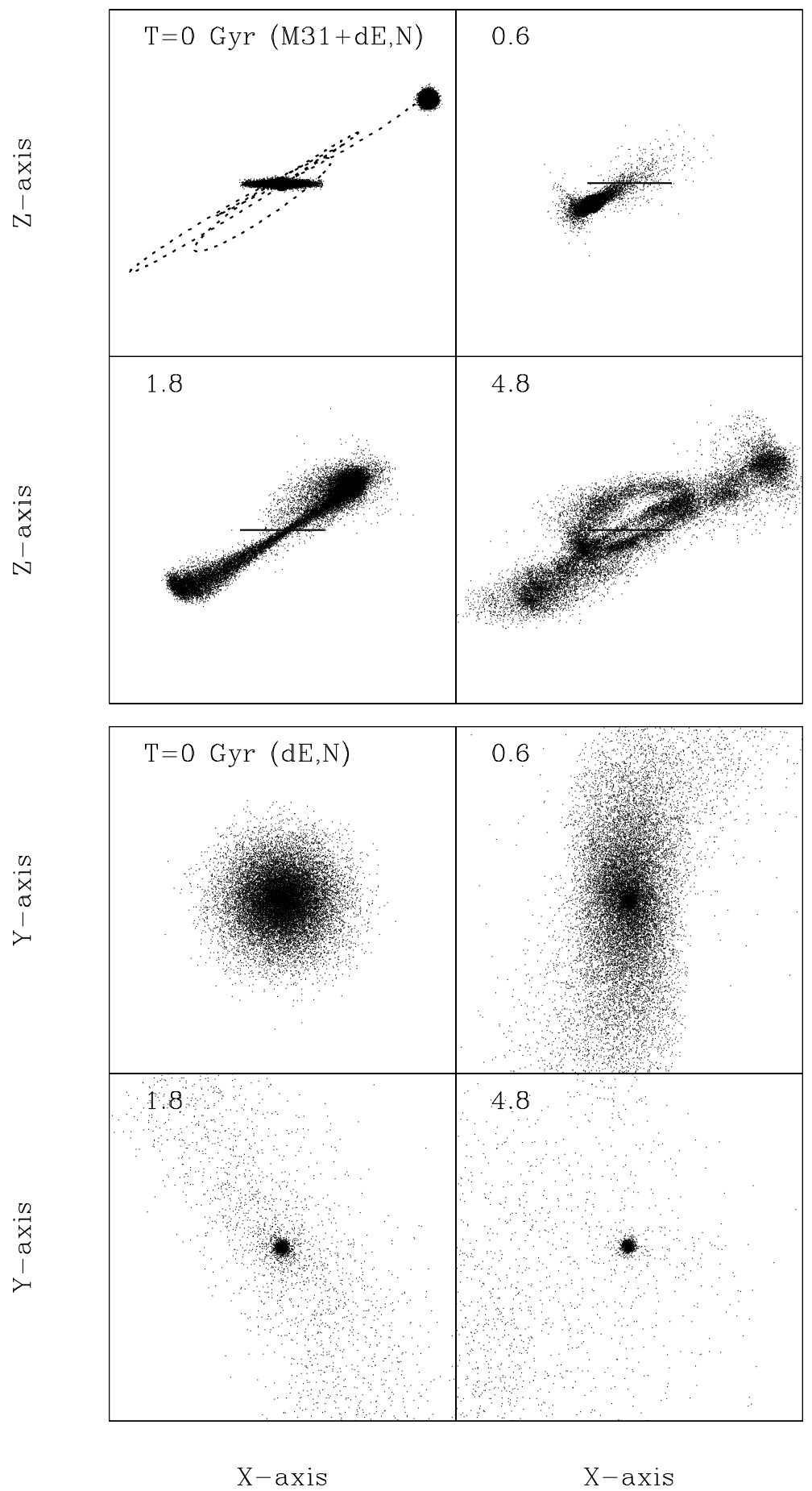

Fig. 1. Upper four: morphological evolution of the stellar envelope of the dE,N projected onto the $x-z$ plane (i.e., edge-on view) for the fiducial model. For comparison, the M 31 disk and bulge components are shown in the upper left panel and the disk size is indicated by a solid line in other three panels. The orbit of the $\mathrm{dE}, \mathrm{N}$ with $R_{\text {apo }}=80 \mathrm{kpc}$ and $e=0.62$ is indicated by a dotted line in the upper left panel. The time $T$ (in units of Gyr) indicated in the upper left corner of each frame represents the time elapsed since the simulation starts. Each frame is $164 \mathrm{kpc}$ on a side. Lower four: morphological evolution of the stellar envelope and the nucleus of the dE,N projected onto the $x-y$ plane (i.e., face-on view) for the fiducial model. Each frame is $20 \mathrm{kpc}$ on a side.

with $\mathrm{M}$ 31. As a result, a very compact stellar system with a negligible amount of dark matter is formed from the dE,N by $T=4.8 \mathrm{Gyr}$. The total nuclear stellar mass of the remnant within $5 a_{\mathrm{dw}}$ is $\sim 1.5 \times 10^{7} M_{\odot}$, consistent with the observed mass of G1. The mass-to-light ratio, $M / L_{\mathrm{B}}$, decreases dramatically from $\sim 10$ to $\sim 3$ for $r<5 a_{\mathrm{dw}}$ within $4.8 \mathrm{Gyr}$. This result clearly explains why $\mathrm{G} 1$ is observed to have mass-to-light ratios that are much smaller than those observed for $\mathrm{dE}$, Ns $(\sim 10)$ : galaxy threshing is most efficient in the outer regions of a $\mathrm{dE}, \mathrm{N}$ where the dark matter halo dominates gravitationally. As is shown in Fig. 2, both the surface density of the dark matter and that of the envelope drop by more than an order of magnitude within 


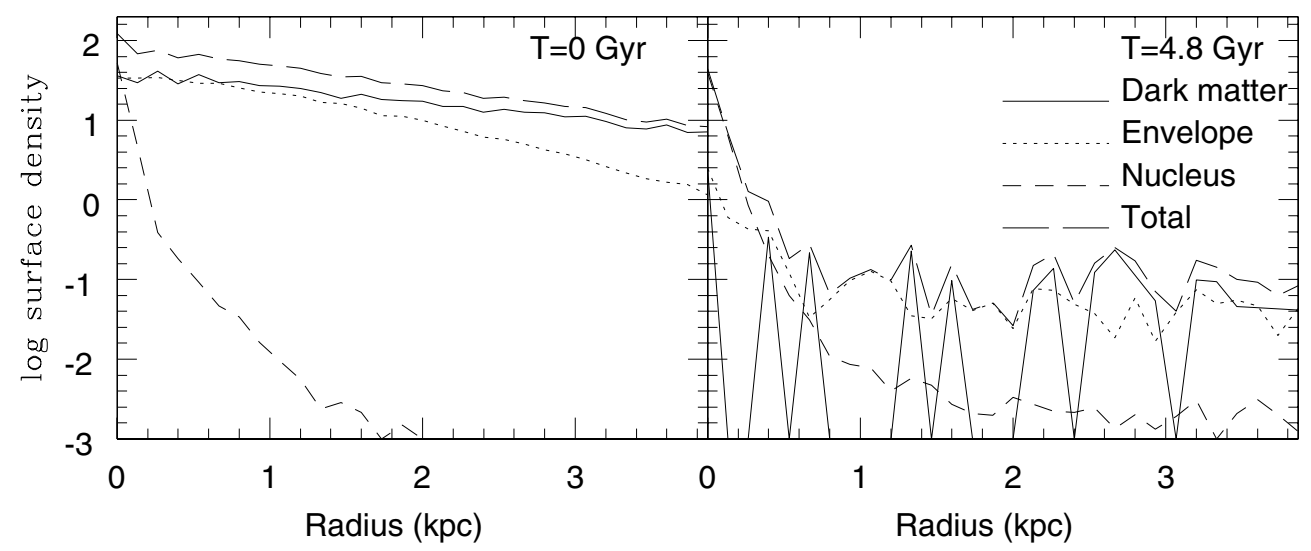

Fig. 2. The projected surface density profiles for the dark matter (solid line), the stellar envelope (dotted line), the nucleus (short-dashed line), and all these components (long-dashed line) at $T=0 \mathrm{Gyr}$ (upper panel) and $4.8 \mathrm{Gyr}$ (lower panel) in the fiducial model.

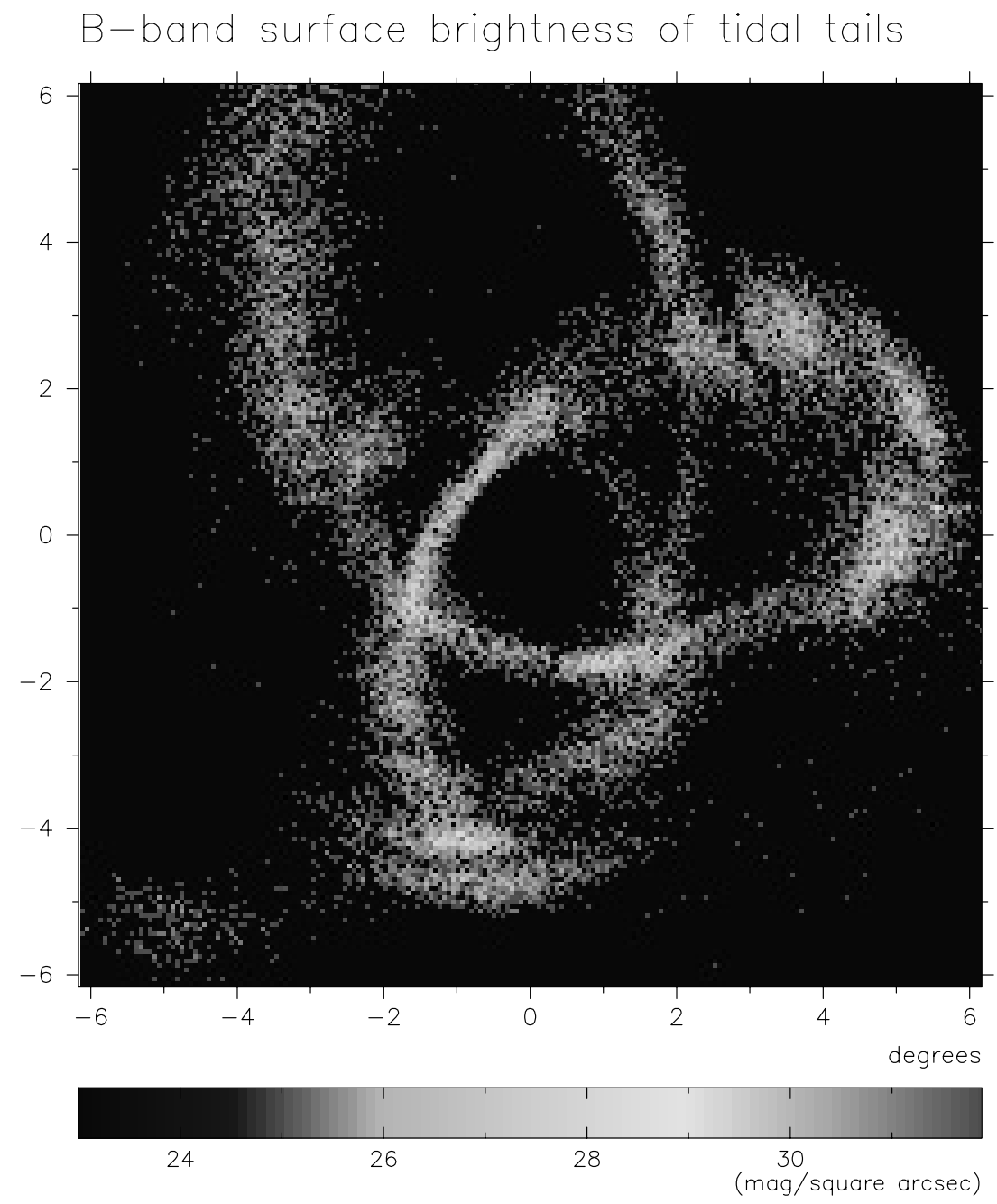

Fig. 3. The final $B$-band surface brightness $\mu_{B}\left(\mathrm{mag} \mathrm{arcsec}^{-2}\right)$ distribution projected onto the $x-y$ plane for substructures/tails of $\mathrm{M} 31$ 's stellar halo developed from tidal destruction of the dE,N in the fiducial model. For comparison with observations by Ferguson et al. (2002), the scale is given in units of degree.

4.8 Gyr. If the initial central surface brightness of the dE, $\mathrm{N}$ is $\mu_{B}=23 \mathrm{mag} \operatorname{arcsec}^{-2}$, then the final surface brightness of the $\mathrm{dE}, \mathrm{N}$ at $r=3 \mathrm{kpc}$ is about $\mu_{B}=29.5 \mathrm{mag} \operatorname{arcsec}^{-2}$. Such a faint, low surface brightness envelope will be hard to detect, even by existing large ground-based telescopes.
The stripped stars from the envelope of the G1 progenitor dE,N form substructures and tidal tails in M 31 halo region. Figure 3 shows that the tidal tail is a "rosette", reflecting the $\mathrm{dE}, \mathrm{N}$ eccentric orbit $\left(e_{\mathrm{p}}=0.62\right)$. Most of the stellar components of the tail can be regarded as being located well 


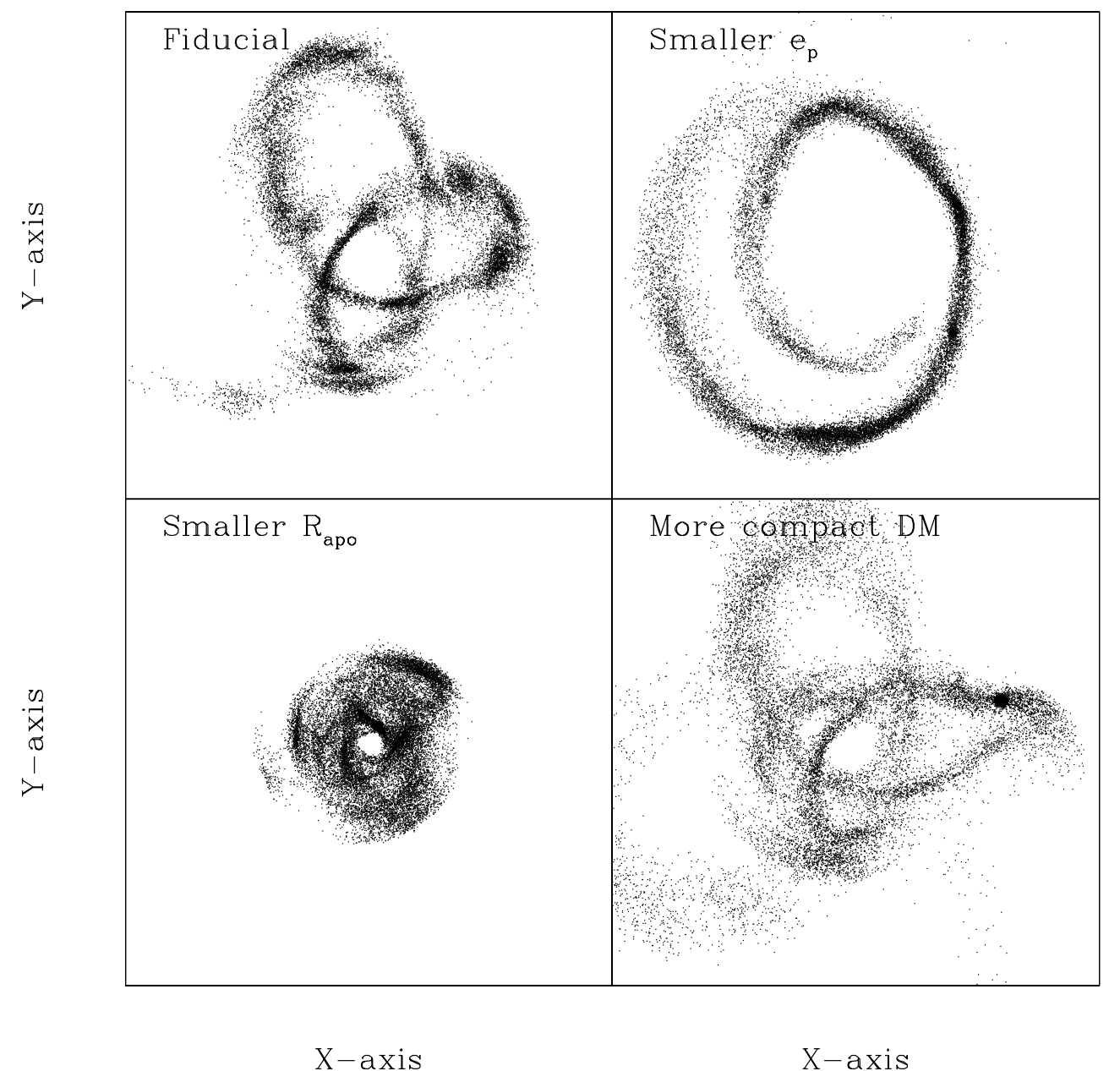

Fig. 4. Final mass distributions projected onto the $x-y$ plane at $T=4.8 \mathrm{Gyr}$ for the fiducial model (upper left), the smaller $e_{\mathrm{p}}$ one with $e_{\mathrm{p}}=0.18$ (upper right), the smaller $R_{\text {apo }}$ with $R_{\text {apo }}=40 \mathrm{kpc}$ (lower left), and the more compact dark matter model with $a_{\mathrm{dm}}=0.25 a_{\mathrm{dm}, 0}($ lower right). Each frame is $240 \mathrm{kpc}$ on a side.

outside the M 31 inner halo with $r<40 \mathrm{kpc}$ ( $\sim$ three degrees). The $B$-band surface brightness $\left(\mu_{B}\right)$ of the tail ranges from $28.7 \mathrm{mag} \operatorname{arcsec}^{-2}$ to $30.4 \mathrm{mag} \operatorname{arcsec}^{-2}$ with a mean of $30.1 \mathrm{mag} \operatorname{arcsec}^{-2}$. These results imply that the tidal streams formed from the $\mathrm{G} 1$ progenitor $\mathrm{dE}, \mathrm{N}$ would be hard to detect in previous observations by Ibata et al. (2001) and Ferguson et al. (2002) that mapped the M 31 halo region within $r \sim 40 \mathrm{kpc}$.

Morphological properties of tails and substructures developed during tidal destruction of a G1 progenitor $\mathrm{dE}, \mathrm{N}$ depend not only on the initial orbit but also on the dark matter structure of the dE,N. Figure 4 summarizes the following three dependences. Firstly, for the model with a smaller $e_{\mathrm{p}}(=0.18)$, in which the time scale of morphological transformation from the $\mathrm{dE}, \mathrm{N}$ into $\mathrm{G} 1$ is much longer than that of the fiducial model, the shape of the developed tail is more like a spiral than a rosette. Secondly, the distribution of stars in the developed substructures for the model with a smaller $R_{\text {apo }}(=40 \mathrm{kpc})$ appears to have a sharp boundary within which the stars can be located in a relatively homogeneous manner. Thirdly, the surface density of stars along the tail in the model with a more compact dark matter halo (with $a_{\mathrm{dm}}=0.25 a_{\mathrm{dm}, 0}$ ) is much lower than that of the fiducial model owing to the smaller number of stripped stars.
Formation of G1 from the dE,N does not occur in this model with $a_{\mathrm{dm}}=0.25 a_{\mathrm{dm}, 0}$ which suggests that a dE,N with the high central density of the dark matter (such as seen in NFW models) are less likely to be a G1 progenitor dE,N (See Bekki et al. 2003 for more discussions on this point).

\section{Discussion and conclusion}

If $\mathrm{G} 1$ originates from a dE,N with $M_{B}=-15 \mathrm{mag}$, the mean metallicity of the M 31 stellar halo consisting of stars stripped from the $\mathrm{dE}, \mathrm{N}$ is roughly estimated as $[\mathrm{Fe} / \mathrm{H}]=$ -0.96 (for $B-V=0.71$ in the $\mathrm{dE}, \mathrm{N}$ ) using the observed metallicity-luminosity relation for dwarf galaxies, $[\mathrm{Fe} / \mathrm{H}]=$ $-3.43( \pm 0.14)-0.157( \pm 0.012) \times M_{V}$ (e.g., Côté et al. 2000) Because of its small mass (less than $10^{9} M_{\odot}$ ) and low metallicity, the stellar halo formed from the tidal destruction of the G1 progenitor $\mathrm{dE}, \mathrm{N}$ could not be the major component of the observed high density and metal-rich $([\mathrm{m} / \mathrm{H}] \sim-0.5)$ M 31' stellar halo (Durrell et al. 2000). Also, relatively metal-rich components $([\mathrm{Fe} / \mathrm{H}] \sim-0.7)$ of the stellar tail recently discovered in the M 31 halo region (Ibata et al. 2001) might be less likely to be formed from the metal-poor stars stripped from G1's progenitor $\mathrm{dE}, \mathrm{N}$. 
Recently Ferguson et al. (2002) discovered a stellar halo substructure located in the proximity of G1 (referred to as "G1 clump") and showed that total $V$-band magnitude and $V$ band surface brightness of the G1 clump can be estimated to be $-12.6 \mathrm{mag}$ and $28.5 \mathrm{mag} \mathrm{arcsec}^{-2}$, respectively, for reasonable assumptions of dust extinction. The present study has demonstrated that metal-poor $([\mathrm{Fe} / \mathrm{H}] \sim-1)$ and low surface brightness $\left(\mu_{B} \sim 30 \mathrm{mag} \operatorname{arcsec}^{-2}\right)$ stellar halo substructures can be formed along the orbit of the G1 progenitor. We have also found that the stars in substructures close to the simulated G1 have orbital properties similar to those of the G1. Accordingly, future spectroscopic observations on radial velocities and metallicities of stars in the G1 clump will provide new clues to the problem of whether the G1 clump can be tidal debris of the G1 progenitor dE,N.

Our simulations suggest that if a dE,N has a higher central dark matter density (i.e., more compact core), it cannot be transformed into a giant globular cluster (G1) because of the survival of its stellar envelope. Whether a $\mathrm{dE}, \mathrm{N}$ can be transformed into a globular cluster by the M 31 tidal field (i.e., by galaxy threshing) depends on whether the M 31 tidal force is stronger than the self-gravitational force of the dark matter halo of the $\mathrm{dE}, \mathrm{N}$ at the pericenter of the orbit of the $\mathrm{dE}, \mathrm{N}$. Therefore we can give the upper limit of the central dark matter density of the G1 progenitor $\mathrm{dE}, \mathrm{N}$, if we know its pericenter distance from M 31. The possible central density of the dark matter halo of $\omega$ Cen's progenitor dE,N is discussed in Bekki \& Chiba (2003), based on the proper motion data of $\omega$ Cen.

Drinkwater et al. (2003) recently discovered a new type of galactic object with effective radius of $\sim 20 \mathrm{pc}, M_{V} \sim-12 \mathrm{mag}$, and velocity dispersion of $\sim 30 \mathrm{~km} \mathrm{~s}^{-1}$ in the Fornax Cluster. These ultra-compact dwarfs (UCDs) with $M_{V}$ more than $1 \mathrm{mag}$ brighter than that of G1 have been demonstrated to be formed by galaxy threshing in which $\mathrm{dE}$,Ns with $M_{V}<-16$ mag can be transformed into UCDs owing to tidal stripping of the stellar envelopes of dE,Ns (Bekki et al. 2001, 2003). We thus suggest that UCDs and giant globular clusters such as G1 and $\omega$ Cen can be regarded as the same class of stellar objects: the total mass or luminosity of a progenitor $\mathrm{dE}, \mathrm{N}$ is the main difference between UCDs and G1. However, the difference in the location on the $M_{V}-\sigma_{0}$ relation between UCD and G1 cannot be explained simply by the galaxy threshing scenario (Bekki et al. 2003).

The present study suggests that if G1 is not close to the pericenter of its orbit around M 31, the stripped stars from the $\mathrm{G} 1$ progenitor $\mathrm{dE}, \mathrm{N}$ can be distributed throughout the M 31 outer halo region with $R_{\mathrm{p}}>40 \mathrm{kpc}$. Therefore, future observations on M 31's stellar halo need to extend to the current limit of previous surveys $\left(R_{\mathrm{p}} \sim 40 \mathrm{kpc}\right.$; Ferguson et al. 2002) to reveal possible tidal streams and substructures formed from dE,N. Metallicity information of the possible streams and substructures in M 31's outer halo is also useful to constrain the stellar population of the destroyed G1 progenitor dE,N. Thus, future deep, high-resolution, wide-area surveys of M 31's outer $\left(R_{\mathrm{p}}>40 \mathrm{kpc}\right)$ stellar halo by wide-field cameras on large ground-based telescopes (e.g., Suprime-Cam on Subaru) will enable us to determine whether G1 originates from an ancient dE,N orbiting M 31 .

Acknowledgements. K.B. acknowledges the Large Australian Research Council (ARC). All the simulations described here were performed with GRAPE 5 systems at the National Astronomical Observatory in Japan.

\section{References}

Bekki, K., Couch, W. J., \& Drinkwater, M. J. 2001, ApJ, 552, L105

Bekki, K., Couch, W. J., Drinkwater, M. J., \& Shioya, Y. 2003, MNRAS, 344, 399

Bekki, K., \& Chiba, M. 2003, in preparation

Bekki, K., \& Freeman, K. C. 2003, MNRAS, accepted

Binggeli, B., \& Cameron, L. M. 1991, A\&A, 252, 27

Burkert, A. 1994, MNRAS, 266, 877

Côté, P., Marzke, R. O., West, M. J., \& Minniti, D. 2000, ApJ, 533, 869

Dinescu, D. I. 2002, in Omega Centauri, A Unique Window into Astrophysics, ed. F. van Leeuwen, J. D. Hughes, \& G. Piotto (San Francisco: ASP), ASP Conf. Ser., 265, 608

Djorgovski, S. G., Gal, R. R., McCarthy, J. K., et al. 1997, ApJ, 474, L19

Drinkwater, M. J., Gregg, M. D., Hilker, M., et al. 2003, Nature, 423, 519

Durrell, P. R., Harris, W. E., \& Pritchet, C. J. 2000, AJ, 121, 2557

Ferguson, A. M. N., Irwin, M. J., Ibata, R. A., Lewis, G. F., \& Tanvir, N. R. 2002, AJ, 124, 1452

Ferguson, H. C., \& Bingelli, B. 1994, A\&ARv, 6, 67

Freeman, K. C. 1993, in The globular clusters-galaxy connection, ed. G. H. Smith, \& J. P. Brodie, ASP Conf. Ser., 48, 608

Gebhardt, K., Rich, R. M., \& Ho, L. C. 2002, ApJ, 578, L41

Gnedin, O. Y., Zhao, H., Pringle, J. E., et al. 2002, ApJ, 568, L23

Hilker, M., \& Richtler, T. 2000, A\&A, 362, 895

Ibata, R., Irwin, M., Lewis, G., Ferguson, A. M. N., \& Tanvir, N. 2001, Nature, 412, 49

King, I. R. 1962, AJ, 67, 471

Meylan, G., Jablonka, P., Djorgovski, S. G., et al. 1997, BAAS, 29, 1367

Meylan, G., Sarajedini, A., Jablonka, P., et al. 2000, BAAS, 32, 1440

Meylan, G., Sarajedini, A., Jablonka, P., et al. 2001, AJ, 122, 830

Mizutani, A., Chiba, M., \& Sakamoto, T. 2003, ApJ, 589, L89

Navarro, J. F., Frenk, C. S., \& White, S. D. M. 1996, ApJ, 462, 563

Rich, R. M., Mighell, K. J., Freedman, W. L., \& Neill, J. D. 1996, AJ, 111,768

Salucci, P., \& Burkert, A. 2000, ApJ, 537, L9

Sugimoto, D., Chikada, Y., Makino, J., et al. 1990, Nature, 345, 33 van den Bergh, S. 2000, The Galaxies of the Local Group (Cambridge, UK: Cambridge University Press), Cambridge Astrophys. Ser., 35

Zhao, H. S. 2002, in Omega Centauri, A Unique Window into Astrophysics, ed. F. van Leeuwen, J. D. Hughes, \& G. Piotto (San Francisco: ASP), ASP Conf. Ser., 265, 391

Zinnecker, H., Keable, C. J., Dunlop, J. S., Cannon, R. D., \& Griffiths, W. K. 1988, in Globular cluster systems in Galaxies, ed. J. E., Grindlay, \& A. G., Davis Philip (Dordrecht: Kluwer), 603 